\title{
Advances in Glaucoma Treatment and Management: Gene Therapy
}

\author{
Terete Borrás
}

G laucoma is characterized by the death of retinal ganglion J cells (RGCs) and loss of vision. It is the second most frequent cause of irreversible blindness in the world and affects primarily the older population. ${ }^{1}$ Projections of prevalence show that by 2020 there will be an estimated 79.6 million people worldwide with glaucoma, with a higher proportion of women than men. ${ }^{1}$ Glaucoma can be treated but is not yet curable. Treatments currently available, such as the daily administration of eye drops, lead to high levels of noncompliance, especially in the aged population. In several laboratories, including ours, intense research is ongoing to search for alternative treatments of the disease. Using genes, we seek to develop gene drug therapies that can be many times more efficient than conventional drugs and allow less frequent administration-perhaps just once every 1 or 2 years. In cases of inherited glaucoma caused by either recessive or dominant genes, we seek gene replacement and gene knockdown strategies that could reverse the undesired outcome of the mutation. Technology for this new state-of-the-art treatment is currently available and has been successfully applied in other diseases, including conditions in the eye. ${ }^{2}$

Most glaucomas are induced by dysfunction of the trabecular meshwork (TM) tissue, which in turn leads to increased resistance to aqueous humor outflow and elevated intraocular pressure (IOP). The mechanism by which elevated IOP damages the posterior RGCs is not fully understood. Whether the elevated IOP first damages the RGC axons and then causes the cell body to die or it damages the cell body first, which then causes the axons to die is a subject of intense deliberation. Although elevated IOP is not the only factor causing RGC death, it is the most common. All currently available drugs that lower IOP slow the progression of all glaucomas, even the form referred to as normal-tension glaucoma.

Because of this particular physiology, there are two main avenues to follow in approaching research in gene therapy for glaucoma. The first entails targeting the RGCs with the goal of promoting their survival and protecting them against glaucomatous insults (neuroprotection). The second entails targeting the TM with the goal of lowering IOP.

Gene therapy vectors deliver the genetic material to the inside of the cells. Because viruses have been selected during evolution to cleanly and efficiently enter living cells, they have emerged as excellent carriers of any desired genetic material. Given the tools of molecular biology and recombinant DNA that are now available, a virus can be specially engineered both to remove the potentially deleterious sequences from its ge-

From the Department of Ophthalmology, University of North Carolina School of Medicine, Chapel Hill, North Carolina.

Submitted for publication January 13, 2012; revised March 4, 2012; accepted March 10, 2012.

Disclosure: T. Borrás, None

Corresponding author: Terete Borrás, Department of Ophthalmology, University of North Carolina, 4109C Neuroscience Research Building, Campus Box 7041, Chapel Hill, NC 27599; tborras@med.unc.edu.

nome and to insert the beneficial foreign sequences to be delivered to the target cell.

\section{Advances in Protection of RGCs by Gene Therapy}

Gene delivery to the RGC is achieved by a single intravitreal injection through the corneal limbus. The viral vector of choice is adeno-associated virus (AAV). This small virus (19-26 $\mathrm{nm}$ ) is nonpathogenic and confers safe and long-term expression of the transgene. It has been used as a vector to deliver the corrected version of the RPE65 gene, which is mutated in animals and humans with Leber's congenital amaurosis, a blinding eye disease. In clinical trials, the gene delivered to the subretinal space by a viral vector restored vision in patients. ${ }^{2}$

\section{Animal Studies of Delivery of Cell Survival Genes to the RGCs}

Because of the vector's proven safety and efficiency, several laboratories have injected AAV2 carrying neurotrophins, antiapoptotic genes, or defense genes, into the eyes of rat models of glaucoma. As early as 2002, Di Polo's laboratory (LebrunJulien and Di Polo ${ }^{3}$ ) in Canada showed that co-injecting AAVs carrying brain-derived neurotrophic factor and its receptor TrkB increased the number of surviving RGCs by $76 \%$. Similar experiments using different rat and mouse models of glaucoma have validated the initial findings. McKinnon et al. ${ }^{4}$ showed that an AAV2 construction carrying the caspase inhibitor baculovirus IAP repeat-containing protein (BIRC4) led to a 53\% survival of optic nerve axons in the rat after chronic exposure to elevated IOP. ${ }^{4}$ In 2009, Quigley, Hauswirth, and coworkers (Pease et al. ${ }^{5}$ ) reported protection of RGC axons by delivering AAV2/ciliary-derived neurotrophic factor to rats. Cao's laboratory (Zhou et al. $^{6}$ ) showed that AAV2/pigment epitheliumderived factor protects the axons of the DBA/2J glaucoma mouse. An important leap in gene therapy protection of RGCs was recently published. Work in Rex's laboratory (Sullivan et al. ${ }^{7}$ ) showed for the first time that systemic administration of a neuroprotective molecule can lead to RGC protection in the DBA/2J glaucoma mouse. The investigators injected AAV2/5 carrying a mutated version of a multiple neuronal protectionencoding gene (erythropoietin R76E; AAV2/5.CMV.EpoR76E) into the quadriceps of the mouse before the animal developed glaucoma. By 10 months of age, when the disease develops in this mouse model, the RGC somata as well as their axons and visual function were protected when compared to controls given AAV2/5 reporter vectors. ${ }^{7}$ In addition, the systemically delivered mutated gene reduced the toxic side effect of the parent gene and maintained normal hematocrit levels (percentage of red blood cell volume). These encouraging findings show that research is leading to less toxic and more patientfriendly administration of neuroprotectants as potential adjunctive therapy for glaucoma. 


\section{New Technology for Longitudinal Evaluation of RGC Survival}

Important advances in imaging technology have allowed for the measurement of the progression of RGC survival in the same animal. Because of the existing significant variation in individual measurements, the relevance of developing imaging methods that observe changes longitudinally in each living animal is very high. Such technologies not only bring a higher degree of reliability and accuracy to animal research but have potential applications in the clinical setting, including the direct counting of fluorescently labeled RGCs using a confocal scanning laser ophthalmoscope (cSLO) and the assessment of the thickness of the nerve fiber layer of the retina using spectral domain optical coherent tomography (SD-OCT). These new, emerging imaging evaluations will, in addition, considerably reduce the number of animals needed for a single study and the cost associated with their maintenance.

\section{Advances in the Treatment of the TM to Lower IOP By GeNe Therapy}

During the past few years, treatment of the TM by gene therapy has shown major progress due to the use of replication-deficient recombinant adenoviral (Ad) vectors. In contrast to the AAV vectors that do not transduce the TM, the Ads have a very high tropism for all cells of the outflow tissue in all animal species tested, from human donor eyes to eyes of monkeys, rabbits, rats, and mice. Although the drawback of the Ad vectors is the limited length of expression of the transgene and their induction of an inflammatory response at high concentrations, the combination of their simple engineering, largecapacity, high-tropism infection of nonreplicating cells and no genome integration makes them ideal for preclinical studies targeting the TM. Ad vectors have allowed the determination of the effect of a transgene in outflow facility and the identification of relevant cellular pathways and of regulatory expression elements. Ad vectors have also allowed the development of new elevated IOP models that do not destroy the trabecular tissue. Gene delivery to the TM is achieved by a single intracameral or intravitreal injection through the corneal limbus.

\section{Preclinical Studies of the Delivery of Target Genes with Adenoviral Vectors}

Identification of Genes and Biological Pathways Involved in IOP Regulation. One of the first genes and pathways identified by the use of Ads was the small GTP-binding protein RhoA. When a dominant-negative RhoA gene was inserted into the Ad vector, it switched the wild-type protein for the mutated, inactive RhoA and reduced the IOP of perfused human eyes in organ cultures. ${ }^{8}$ The involvement of this pathway, where the activation of RhoA leads to the activation of Rho kinase (ROCK) and ends in the reorganization of the actin cytoskeleton, has been independently sought and validated in numerous laboratories. ${ }^{9}$ Presently, development of chemical RhoA pathway inhibitors (specifically of ROCK) is being pursued by several pharmaceutical companies as potential drugs for glaucoma. The development of a gene therapy drug carrying the inhibitor gene is likely to come later, after the regulatory elements that control gene expression have been fully worked out.

The use of recombinant Ads has further contributed to the identification of two other TM cellular pathways relevant to glaucoma: the presence of an ongoing calcification process and the involvement of the Wnt signaling pathway. We have shown that an Ad virus carrying the inhibitor of calcification matrix Gla gene (Adh.MGP) inhibits bone morphogenetic 2 (BMP2)- induced mineralization in TM cells. ${ }^{10}$ For the involvement of the WNT signaling pathway, Clark's group (Wang et al. ${ }^{11}$ ) injected an Ad virus carrying the gene encoding the WNT antagonist secreted frizzled-related protein 1 (SFRP1) in the mouse eye. SFRP1 induced an elevation of pressure that was reversed by the administration of the inhibitor GSK. The existence of these two pathways in TM physiology opens the door to the development of new therapeutic targets for glaucoma. Thus, replenishing a low MGP expression during early glaucoma or helping restore a functional WNT pathway could delay the progress of the disease.

Insertion of Regulatory Elements for Expressing Therapeutic Genes Only When Needed. In a gene therapy regimen in which genes serve as drugs, a system must be in place that allows the expression of the gene only when it is needed. Advances have been made on vectors carrying inducible regulatory sequences in the promoter of potentially therapeutic genes. In the most successful study to date, an Ad vector developed for the treatment of steroid glaucoma carried the matrix metallopeptidase 1 (MMP1) gene regulated by glucocorticoid response elements (Adh.GRE.MMP1). Intracameral injection of this vector in a model of steroid glaucoma in sheep not only reduced elevated IOP but also prevented its elevation when injected 2 days before the steroid was applied (Fig. 1). ${ }^{12,13}$ This coupled study has provided the first in vivo proof of concept and has established the basis for further development of this treatment.

Other studies using pressure-regulatory elements as well as elements that confer TM tissue preference in Ad vectors are in the pipeline and have been reported at recent scientific meetings. The promoter of the MGP gene has been fused to the gene encoding secreted alkaline phosphatase and perfused into human donor eye cultures. Induction of elevated pressure in one of the two paired eyes resulted in higher secretion of the reporter enzyme (Carabana J, et al. IOVS 2011;52:ARVO E-Abstract 4623). This finding indicates that the MGP promoter elements respond to elevated IOP. Therefore, these sequences could be inserted in front of different therapeutic genes, regulating them to produce their therapeutic protein only when IOP is high. In another study, the promoter of the $\alpha \mathrm{B}$-crystallin gene was fused to a reporter gene that, when expressed, turns blue through an enzyme reaction, thus making it easy to detect in the tissue. This promoter delivered its transgene to a particular region of the TM known for its relevance in offering resistance to the flow of eye fluid, the juxtacanalicular region (Spiga M, et al. IOVS 2008; 49:ARVO E-Abstract 1612). ${ }^{14}$ Elucidation of the parameters that control gene expression will make possible the application of gene therapy technology in the treatment of glaucoma.

Generation of Animal Models of Elevated IOP by Ad Gene Transfer. Another important advance in work on Ad gene delivery to the TM has been the generation of elevated IOP animal models for the evaluation of conventional and/or gene therapy drugs. Most of today's animal models of elevated IOP glaucoma are obtained by scarring or destroying the TM tissue by different means. Although such models are adequate for the study of neuroprotection of the RGCs, they are of no use in studying therapeutic targeting of the TM. We reasoned that delivering genes that produce a thicker, denser extracellular matrix would slow the flow of the eye fluid when exiting the eye through the TM. We found that a single injection of an Ad vector carrying the mineralization protein BMP2 into the anterior chamber of rats significantly induced an elevation of IOP for at least 6 weeks after injection (Buie L, et al. IOVS 2009;50:ARVO E-Abstract 4841). Another Ad virus carrying the extracellular matrix deposition stimulator and known glaucoma-associated factor, transforming growth factor beta 2 (Ad.hTGF $\beta 2$ ), also elevated IOP in rodents. ${ }^{15}$ The IOP increase 

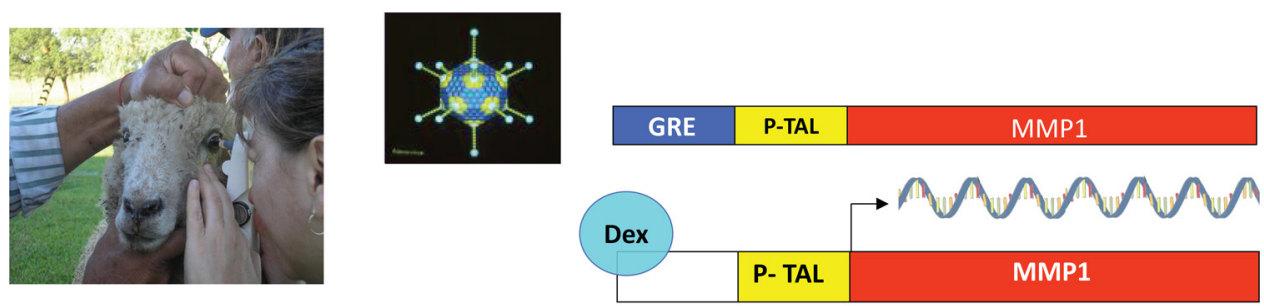

\begin{tabular}{|c|c|c|c|}
\hline \multicolumn{4}{|c|}{ Sheep 378 , os } \\
\hline Day & Drug & $\begin{array}{c}\text { Viral } \\
\text { Injection }\end{array}$ & $\begin{array}{l}\text { IOP } \\
\mathrm{mmHg}\end{array}$ \\
\hline 0 & Prednisolone & & 13.7 \\
\hline 1 & Prednisolone & & 11.6 \\
\hline 2 & Prednisolone & & 22.3 \\
\hline 3 & Prednisolone & Adh.GRE.MMP1 & 23.6 \\
\hline 5 & Prednisolone & & 9.4 \\
\hline 9 & Prednisolone & & 9.4 \\
\hline 15 & Prednisolone & & 11.6 \\
\hline 24 & Prednisolone & & 22.3 \\
\hline
\end{tabular}

\begin{tabular}{|c|c|c|c|}
\hline \multicolumn{4}{|c|}{ Sheep 377 , os } \\
\hline Day & Drug & $\begin{array}{c}\text { Viral } \\
\text { Injection }\end{array}$ & $\begin{array}{c}\text { IOP } \\
\mathrm{mmHg}\end{array}$ \\
\hline 0 & & Adh.GRE.MMP1 & 13.7 \\
\hline 1 & & & 11.6 \\
\hline 2 & & & 13.7 \\
\hline 3 & Prednisolone & & 10.7 \\
\hline 5 & Prednisolone & & 11.6 \\
\hline 9 & Prednisolone & & 12.9 \\
\hline 15 & Prednisolone & & 9.4 \\
\hline 24 & Prednisolone & & 21.5 \\
\hline
\end{tabular}

\section{Reduction of steroid-induced elevated IOP in sheep treated with gene therapy vector Adh.GRE.MMP1}

FigURE 1. Reduction of steroidinduced elevated IOP in sheep treated with gene therapy vector Adh.GRE.MMP1. occurred only when a bioactivated, mutated form of TGF $\beta 2$, rather than the wild type, was delivered; the effect lasted for approximately 3 weeks. The investigators also showed a correlation between the increase in IOP and a decrease in outflow facility, thus clearly implicating the TM. The success of this strategy of creating elevated IOP models by gene transfer, now validated in two different laboratories, is going to have great impact in the near future for gene therapy. It will open the door, not only to different types of models, but to the opportunity of generating large animal models consecutively-that is, the possibility of using the same large animal for the generation of different models.

\section{Advances Using Additional Gene Delivery Approaches}

\section{Lentiviral Vectors for Glaucoma Gene Therapy}

Lentiviral vectors are derived from immunodeficiency viruses. The available vectors have originated from human, simian, and feline viruses (HIV, SIV, and FIV) and are depleted of potentially dangerous virus replication genes. Although somewhat more difficult to work with because of low titers and psychological concerns, these vectors deliver transgenes to the RGCs and TM very efficiently. ${ }^{16}$ They are single-stranded RNA viruses, and, on infection, they integrate the cell's DNA by reverse transcription. The immediate consequences of this property are the possibilities of disrupting a vital gene by random insertion and of obtaining long-term expression of the transgene. A very recent example of an SIV vector carrying the neuroprotective molecule human PEDF and injected subretinally into rats showed protection of the number of RGCs and of their function in two models of glaucoma. ${ }^{17}$ In normotensive cats, FIV vectors carrying different prostaglandin pathway enzymes with optimized codons were co-injected intracamerally. Injections of single vectors did not have much effect on IOP, but the combination cyclooxigenase- 2 with other prostaglandin pathway enzymes induced and maintained an IOP reduction for more than 5 months. ${ }^{16,18}$

\section{Delivery of Nonviral Short-Interference (si)RNA and Nanoparticles}

An alternative gene therapy approach without the use of viralbased vectors entails the use of siRNA-short (21 nt), doublestranded RNA oligonucleotide chains that can silence the translation of a specific mRNA. These specific sequences targeting a given gene can be determined by using bioinformatics tools and can be synthesized in a laboratory. The goal of delivering siRNA to a cell or tissue is to silence an unwanted gene temporarily. In general, an siRNA molecule needs some help to enter the cell. Thus, a concerted research effort is ongoing in the area of nanotechnology that aims to increase and target drug delivery to the eye. Nanoparticles of different polymer compositions, including magnetic nanoparticles, are being coupled to siRNAs (as well as to genes, antibodies, and peptide factors) to facilitate entrance. These nanoparticles are being delivered to the front and back of the eye and assayed for functional delivery. ${ }^{19}$ A few studies, however, have demonstrated that a naked siRNA molecule can, by itself, penetrate human TM tissue when perfused at physiological pressure. ${ }^{20}$ In our laboratory, perfusion of the glucocorticoid receptor naked siRNA not only silenced this gene but blocked the induction of other downstream genes induced by glucocorticoids in the TM. ${ }^{20}$ Continuation experiments to deliver naked siRNA to the TM in a living animal have been encouraging. At the last ARVO annual meeting, a study was presented in which topical administration of a commercial naked siRNA lowered IOP in clinical trials (Ruz V, et al. IOVS 2011:52 ARVO E-Abstract 223).

\section{Preparing to Target the TM with Clinically Tested Vectors}

To date, there have been 12 clinical trials using gene therapy viral vectors for the treatment of ocular diseases in the United States and less than 5 worldwide (Journal of Gene Medicine Clinical Trial database: http://www.abedia.com/wiley/index. html; updated January, 2012). Of the 12 U.S. studies, 6 involved AAV2 vectors. The greatest success has been achieved with the replacement of the RPE65 wild-type gene in blind patients 
with Leber's congenital amaurosis who carry a mutation in the gene. ${ }^{21,22}$ The AAV2.RPE65 was administered by subretinal injection into a single eye, and patients showed significant vision improvement in dim light with no safety issues. Efficacy and safety have been sustained for up to 4 years and readministration of the same virus to the second eye of three adult patients resulted in improvement of retinal visual function and visual cortex activation. ${ }^{21}$ This important success has in itself validated the use of AAV viral vectors in gene therapy for the eye. AAVs transduce the RGCs very efficiently, but as mentioned, they do not transduce the TM. Studies to elucidate the cellular mechanism responsible for such lack of transduction led us to the discovery that, in contrast to what we originally thought, the virus did not have any problem entering the TM cell. The limitation occurs one step downstream of the infection cycle, where the virus first replicates its single-stranded DNA and then transcribes and translates the transgene. ${ }^{23}$ The development at the University of North Carolina Gene Therapy Center of a second-generation AAV-self-complementary (scAAV) - that circumvents the DNA replication-limiting step, now allows safe and long expression in the TM. A single injection of an scAAV2.GFP into a living monkey conferred stable expression of the transgene for 2 years without eliciting an inflammatory response. ${ }^{24}$

We thus have a clinical trial-tested vector for targeting the main tissues involved in the development of glaucoma: the TM and the RGCs. This puts us in a very favorable position to be able to insert a therapeutic transgene in this vector and take it to a clinical trial within a reasonable time frame.

\section{Advances in Identifying Gene Targets for LOWERING IOP}

Glaucoma is a complex disease that is probably caused by several genetic factors. Some of these factors are highly penetrant and have been detected through family-based genetic linkage studies, whereas others have more modest overall effect sizes and have been identified through large-scale wholegenome association studies. Currently, 16 chromosomal loci have been mapped, and four POAG genes have been identified by linkage analysis. ${ }^{25}$ In addition, more than 25 genes have been identified by candidate gene and gene association studies. $^{26}$ The most studied highly penetrant glaucoma gene is myocilin (a TM-inducible glucocorticoid response gene). ${ }^{27}$ There are more than $70 M Y O C$ mutations, all associated with elevated IOP, ranging from mild to severe (http://myocilin.com/; reviewed in Ref. 28). Myocilin mutations (albeit not a single one) account for $\sim 36 \%$ of cases of the juvenile form of the disease, but for only $\sim 3 \%$ of adult cases (http://myocilin$. \mathrm{com} /$ ). Some, such as $\mathrm{Gln} 368 \mathrm{X}$, are typically associated with adult-onset POAG, ${ }^{29}$ whereas others, such as Pro370Leu, are nearly always associated with early onset. ${ }^{30}$ Because myocilin mutations are autosomal dominant and most likely cause the disease by a gain-of-function mechanism, the prospect of gene therapy for a patient with a myocilin mutation would be a combined gene knockdown and gene addition therapy. That would involve the use of siRNA to block the mRNA of the mutant allele and an overexpressed normal gene to boost the levels of only one normal allele. It could be considered for the treatment of a young patient whose high IOP would be a considerable burden for the rest of his or her life and who would have a significant chance of become blind. Recent advances in the generation of transgenic mice with myocilin mutations and elevated pressure could permit the development of an assay to assess the safety and efficacy of such vectors in gene therapy.

In addition to the few genetically linked genes, numerous sequencing and gene profile studies have identified a set of 50 potentially relevant genes that we have termed the biomarkers or the molecular signature of glaucoma in the TM. ${ }^{31}$ These genes have been identified in different laboratories, either as highly abundant in the human tissue or highly regulated by glaucomatous insults to the TM. ${ }^{31}$ They provide a starting point for the development of gene therapy drugs to lower IOP. A perfused organ culture comprehensive study involving human genes whose expression is altered by elevated IOP revealed that both an individual and a general response were present in the human eye. ${ }^{32}$ General responder genes, such as MMP1, angiopoietin-like 7, MGP, podoplanin, and the chemokine CXCL $2,{ }^{32}$ are examples of good gene therapy candidates. A cross-checked correlation of seven glaucoma-related categories (gene abundance, response to glucocorticoids, TGF $\beta 2$, mechanical strain, glaucoma tissue sample transcription and proteomics, and alteration by myocilin) stratified the biomarker list by the number of insults that affected a given gene. These categories may well be a representation of the genes most likely to succeed in a gene therapy effort. The encouraging results mentioned earlier from the studies of an $M M P 1$ viral vector lowering elevated IOP in sheep ${ }^{12,13}$ confer a degree of confidence regarding the success of the project.

\section{SUMMARY}

The first question that can be posed is whether we are ready to take a gene therapy vector to clinical trial for lowering IOP and for glaucoma treatment. A recap of what we have and what we need in order to achieve our goal leads to a seven-point plan on how to proceed:

1. Make a decision about the type of glaucoma to treat. There are several types of glaucoma: primary (including juvenile) open-angle glaucoma, congenital, pseudoexfoliation, and steroid. We cannot address all of these at the same time. We will achieve better results if we focus first on a particular type of glaucoma.

2. Selection of targets. Regardless of whether we are going to try the gene knockdown/addition route or the gene therapy drug approach, we should evaluate and select which mutation or which genes (one- to two-gene drug) we will develop first. We have good candidates.

3. Selection of vector pseudotype and route of administration. Pseudotyping of the same virus or the ability to incorporate different targeting proteins in its envelope can significantly affect the tropism of the virus for a given cell type. Now that the general safety of the AAV vector has been established in the human (so far, up to 4 years), ${ }^{2,21}$ a few pretrial studies are needed to determine which of the various virus pseudotypes would be more specific for the TM. Further, the route of administration for the TM, whether by intravitreal or intracameral injection, must be validated.

4. Control of transgene expression by gene regulatory elements. A long-term constitutive expression of a gene inside a cell may not always be beneficial. The ideal gene drug should have built-in signals that would allow a gene to be turned on and off as needed. Ideally, these signals would be automatic - that is, directly switched on by a glaucomatous insult. They could also be induced by the oral intake of the inducer.

5. Reliable animal efficacy evaluation procedures. Assays to determine the effects of gene delivery as well as visual function in animal models are critical. Fortunately, the past 5 years have produced several instruments that assess, for the first time, the physiological parameters and noninvasive imaging morphometric determinations in rodent eyes. Among them are the rebound tonometer, 
a very recent aqueous humor dynamics apparatus ${ }^{33}$; a pattern electroretinogram to measure function in RGCs; visual evoked potentials; and optical coherence tomography.

6. Validation in different animal models. Testing of the effect of the gene therapy vector, as with any conventional drug, would require the confirmation of the effect in more than one animal species. Again, during the past 2 to 3 years, the availability of TM animal models has been improving. There are new strategies for using gene transfer (BMP2 or TGF $\beta 2$ genes) or microbeads in the creation of an animal model of elevated pressure that does not destroy the TM. The advantage of these strategies is the possibility of generating large animal models without the expense required in the creation of transgenic animals

7. Validation in different laboratories. It is essential that researchers in different laboratories independently validate results.

From the discussion of this plan by the panel of glaucoma investigators emerged the idea of forming a consortium among a few centers with the focused goal of moving toward the treatment of glaucoma by gene therapy.

\section{References}

1. Quigley HA, Broman AT. The number of people with glaucoma worldwide in 2010 and 2020. Br J Opbthalmol. 2006;90:262-267.

2. Maguire AM, High KA, Auricchio A, et al. Age-dependent effects of RPE65 gene therapy for Leber's congenital amaurosis: a phase 1 dose-escalation trial. Lancet. 2009;374:1597-1605.

3. Lebrun-Julien F, Di Polo A. Molecular and cell-based approaches for neuroprotection in glaucoma. Optom Vis Sci. 2008;85:417424.

4. McKinnon SJ, Lehman DM, Tahzib NG, et al. Baculoviral IAP repeat-containing- 4 protects optic nerve axons in a rat glaucoma model. Mol Ther. 2002;5:780-787.

5. Pease ME, Zack DJ, Berlinicke C, et al. Effect of CNTF on retinal ganglion cell survival in experimental glaucoma. Invest Ophthalmol Vis Sci. 2009;50:2194-2200.

6. Zhou X, Li F, Kong L, Chodosh J, Cao W. Anti-inflammatory effect of pigment epithelium-derived factor in DBA/2J mice. Mol Vis. 2009; 15:438-450.

7. Sullivan TA, Geisert EE, Hines-Beard J, Rex TS. Systemic adenoassociated virus-mediated gene therapy preserves retinal ganglion cells and visual function in DBA/2J glaucomatous mice. Hum Gene Ther. 2011;22:1191-1200.

8. Vittitow JL, Garg R, Rowlette LL, Epstein DL, O'Brien ET, Borrás T. Gene transfer of dominant-negative RhoA increases outflow facility in perfused human anterior segment cultures. Mol Vis. 2002;8:32- 44

9. Tanihara $\mathrm{H}$, Inatani M, Honjo M, Tokushige $\mathrm{H}$, Azuma J, Araie M Intraocular pressure-lowering effects and safety of topical administration of a selective ROCK inhibitor, SNJ-1656, in healthy vol unteers. Arch Opbthalmol. 2008;126:309-315.

10. Xue W, Wallin R, Olmsted-Davis EA, Borrás T. Matrix GLA protein function in human trabecular meshwork cells: inhibition of BMP2induced calcification process. Invest Ophthalmol Vis Sci. 2006;47: 997-1007.

11. Wang WH, McNatt LG, Pang $\mathrm{IH}$, et al. Increased expression of the WNT antagonist sFRP-1 in glaucoma elevates intraocular pressure. J Clin Invest. 2008;118:1056-1064.

12. Spiga MG, Borrás T. Development of a gene therapy virus with a glucocorticoid-inducible MMP1 for the treatment of steroid glaucoma. Invest Opbthalmol Vis Sci. 2010;51:3029-3041.
13. Gerometta R, Spiga MG, Borrás T, Candia OA. Treatment of sheep steroid-induced ocular hypertension with a glucocorticoid-inducible MMP1 gene therapy virus. Invest Ophthalmol Vis Sci. 2010; 51:3042-3048.

14. Tamm ER, Russell P, Johnson DH, Piatigorsky J. Human and monkey trabecular meshwork accumulate alpha B-crystallin in response to heat shock and oxidative stress. Invest Ophthalmol Vis Sci. 1996;37:2402-2413.

15. Shepard AR, Millar JC, Pang IH, Jacobson N, Wang WH, Clark AF. Adenoviral gene transfer of active human transforming growth factor- $\beta 2$ elevates intraocular pressure and reduces outflow facility in rodent eyes. Invest Ophthalmol Vis Sci. 2010;51:2067-2076.

16. Balaggan KS, Ali RR. Ocular gene delivery using lentiviral vectors. Gene Ther. 2012;19:145-153.

17. Miyazaki M, Ikeda Y, Yonemitsu Y, et al. Pigment epitheliumderived factor gene therapy targeting retinal ganglion cell injuries: neuroprotection against loss of function in two animal models Hum Gene Ther. 2011;22:559-565.

18. Barraza RA, McLaren JW, Poeschla EM. Prostaglandin pathway gene therapy for sustained reduction of intraocular pressure. $\mathrm{Mol}$ Ther. 2010;18:491-501.

19. Raju HB, Goldberg JL. Nanotechnology for ocular therapeutics and tissue repair. Expert Rev Ophthalmol. 2008;3:431-436.

20. Comes N, Borrás T. Functional delivery of synthetic naked siRNA to the human trabecular meshwork in perfused organ cultures. Mol Vis. 2007;13:1363-1374.

21. Bennett J, Ashtari M, Wellman J, et al. AAV2 Gene therapy readministration in three adults with congenital blindness. Sci Transl Med. 2012;4:120ra15.

22. Jacobson SG, Cideciyan AV, Ratnakaram R, et al. Gene therapy for leber congenital amaurosis caused by RPE65 mutations: safety and efficacy in 15 children and adults followed up to 3 years. Arch Ophthalmol. 2012;130:9-24.

23. McCarty DM, Monahan PE, Samulski RJ. Self-complementary recombinant adeno-associated virus (scAAV) vectors promote efficient transduction independently of DNA synthesis. Gene Ther. 2001;8:1248-1254.

24. Buie LK, Rasmussen CA, Porterfield EC, et al. Self-complementary AAV virus (scAAV) safe and long-term gene transfer in the trabecular meshwork of living rats and monkeys. Invest Ophthalmol Vis Sci. 2010;51:236-248.

25. Pasutto F, Keller KE, Weisschuh N, et al. Variants in ASB10 are associated with open-angle glaucoma. Hum Mol Genet. 2012;21: 1336-1349.

26. Fuse N. Genetic bases for glaucoma. Toboku J Exp Med. 2010;221: $1-10$.

27. Polansky JR, Kurtz RM, Fauss DJ, Kim RY, Bloom E. In vitro correlates of glucocorticoid effects on intraocular pressure. In: Krieglstein GK, ed. Glaucoma Update IV. Berlin, Heidelberg: Springer-Verlag; 1991:20-29.

28. Gong G, Kosoko-Lasaki O, Haynatzki GR, Wilson MR. Genetic dissection of myocilin glaucoma. Hum Mol Genet. 2004;13 Spec No 1:R91-R102.

29. Wiggs JL, Allingham RR, Vollrath D, et al. Prevalence of mutations in TIGR/Myocilin in patients with adult and juvenile primary openangle glaucoma. Am J Hum Genet. 1998;63:1549-1552.

30. Alward WL, Fingert JH, Coote MA, et al. Clinical features associated with mutations in the chromosome 1 open-angle glaucoma gene (GLC1A). N Engl J Med. 1998;338:1022-1027.

31. Borrás T. What is functional genomics teaching us about intraocular pressure regulation and glaucoma. In: Civan MM, ed. The Eye's Aqueous Humor. 2nd ed. San Diego: Elsevier; 2008:323-377.

32. Comes $\mathrm{N}$, Borrás $\mathrm{T}$. Individual molecular response to elevated intraocular pressure in perfused postmortem human eyes. Pbysiol Genomics. 2009;38:205-225.

33. Millar JC, Clark AF, Pang IH. Assessment of aqueous humor dynamics in the mouse by a novel method of constant-flow infusion. Invest Ophthalmol Vis Sci. 2011;52:685-694. 